\section{(6) OPEN ACCESS}

\title{
Perinatal outcomes for extremely preterm babies in relation to place of birth in England: the EPICure 2 study
}

\author{
N Marlow, ${ }^{1}$ C Bennett, ${ }^{1}$ E S Draper, ${ }^{2}$ E M Hennessy, ${ }^{3}$ A S Morgan, ${ }^{1}$ K L Costeloe ${ }^{4,5}$
}

- Additional material is published online only. To view please visit the journal online (http://dx.doi.org/10.1136/ archdischild-2013-305555).

${ }^{1}$ Academic Neonatology, UCL Institute for Women's Health, London

${ }^{2}$ Department of Health Sciences, University of Leicester, Leicester, UK ${ }^{3}$ Wolfson Institute of Preventive Medicine, Queen Mary University of London, London, UK

${ }^{4}$ Centre For Paediatrics, Blizard Institute, Barts and the London School of Medicine and Dentistry, Queen Mary University of London, London, UK

${ }^{5}$ Homerton University Hospital, NHS Foundation Trust, London, UK

\section{Correspondence to} Professor Neil Marlow, UCL EGA Institute for Women's Health, 74 Huntley Street, London WC1E 6AU, UK; n.marlow@ucl.ac.uk

Received 27 October 2013 Revised 19 January 2014 Accepted 26 January 2014 Published Online First 6 March 2014

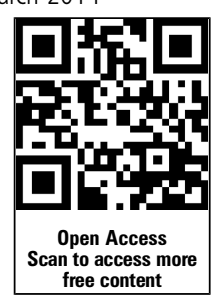

\section{SLinked}

- http://dx.doi.org/10.1136/ archdischild-2014-306020

- http://dx.doi.org/10.1136/ archdischild-2014-306021

CrossMark

To cite: Marlow N, Bennett C, Draper ES, et al. Arch Dis Child Fetal Neonatal Ed 2014;99: F181-F188.
ABSTRACT
Background Expertise and resources may be important determinants of outcome for extremely preterm babies. We evaluated the effect of place of birth and perinatal transfer on survival and neonatal morbidity within a prospective cohort of births between 22 and 26 weeks of gestation in England during 2006.

Methods We studied the whole population of 2460 births where the fetus was alive at the admission of the mother to hospital for delivery. Outcomes to discharge were compared between level 3 (most intensive) and level 2 maternity services, with and without transfers, and by activity level of level 3 neonatal unit; ORs were adjusted for gestation at birth and birthweight for gestation (adjusted ORs (aOR)).

Findings of this national birth cohort, $56 \%$ were born in maternity services with level 3 and $34 \%$ with level 2 neonatal units; $10 \%$ were born in a setting without ongoing intensive care facilities (level 1). When compared with level 2 settings, risk of death in level 3 services was reduced (aOR $0.73(95 \% \mathrm{Cl} 0.59$ to 0.90$)$ ), but the proportion surviving without neonatal morbidity was similar (aOR 1.27 (0.93 to 1.74)). Analysis by intended hospital of birth confirmed reduced mortality in level 3 services. Following antenatal transfer into a level 3 setting, there were fewer intrapartum or labour ward deaths, and overall mortality was higher for those remaining in level 2 services (aOR 1.44 (1.09 to 1.90)). Among level 3 services, those with higher activity had fewer deaths overall (aOR 0.68 (0.52 to 0.89$)$ ).

Interpretation Despite national policy, only $56 \%$ of births between 22 and 26 weeks of gestation occurred in maternity services with a level 3 neonatal facility. Survival was significantly enhanced following birth in level 3 services, particularly those with high activity; this was not at the cost of increased neonatal morbidity.

\section{INTRODUCTION}

Survival and later neurological and developmental outcomes for extremely premature babies remain of concern because of significant mortality and morbidity. ${ }^{1}{ }^{2}$ In the UK, neonatal care services have developed largely in response to local needs, ${ }^{3}$ in contrast to practice in many other countries, where highly regionalised systems have developed. ${ }^{4}$ In 2003 , there was a nationally commissioned review ${ }^{5}$ that subsequently led to the development of managed neonatal clinical networks in England and increased centralisation of care for babies born at 26 weeks of gestation or less. However, there has been a paucity of data from the UK examining the relationship of birth at extremely low gestational ages to the expertise in the hospital of birth and a

\section{What is already known on this topic}

- International data suggest that survival for babies admitted for neonatal intensive care is improved in regional centres.

- Previous data are difficult to interpret because of the confounding effect of antenatal and postnatal transfer.

\section{What this study adds}

- Survival is greater in specialist hospitals in England providing neonatal intensive care and is further improved in higher activity services.

- This improvement is primarily achieved by a reduction in fetal deaths before delivery, and neonatal deaths in the delivery room and over the first week.

- Women who book for their care at specialist hospitals have lower mortality compared with those booking at local (non-specialist) hospitals, although antenatal transfer does result in a group of babies with improved survival chances.

continuing reluctance among practitioners to support the principle that centralisation of specialist services will improve outcomes for this group within the UK model of care. ${ }^{6}$ Thus, it is important to assess the impact that the development of neonatal networks may have had on outcome for this particular group.

In 2006, we carried out a national population study of births in England between 22 and 26 completed weeks of gestation (EPICure 2), from which survival and short-term morbidity data and outcome at 3 years of age have been published. ${ }^{12}$ This report evaluates the hypothesis that birth in, or transfer to, designated referral centres is accompanied by lower rates of mortality and morbidity among survivors.

\section{POPULATION}

Details of all births reported between 22 and 26 weeks' gestation were collected prospectively in all 182 maternity hospitals in England during 2006. Births to mothers not usually resident in England were excluded. This study was based on 
the complete population of mothers with a live fetus at the point of the hospital admission that led to delivery.

\section{METHODOLOGY}

Information was collected on place of maternal booking and delivery for all births, together with details for all livebirths of neonatal transfers and major neonatal morbidities to discharge from hospital. Further details on the neonatal data collection process and population outcomes have been reported previously. ${ }^{1}$

For this report, a 'service' comprises the maternity service and its associated neonatal unit, categorised by level of neonatal unit, using the agreed local designation of each neonatal unit (level 1, 2 or 3), based on guidance in the 2003 national review. Level 1 services were hospitals with a level 1 neonatal unit and would aim to transfer out any woman whose baby was expected to need intensive care; level 2 services would generally aim to transfer out women expected to deliver before 27 weeks of gestation; level 3 services would provide the whole range of medical neonatal intensive care.

We further categorised level 3 neonatal units based on activity data collected by questionnaire, based on that of the United Kingdom Neonatal Staffing Study (UKNSS), ${ }^{7}$ sent during the autumn of 2006 to named EPICure contacts in each hospital. The EPICure perinatal steering group categorised units into high activity ( $\geq 2000$ days respiratory support (ventilation or continuous positive airways pressure) per year and more than four consultants with more than $50 \%$ of their time dedicated to neonatology), medium activity (500-1999 days of respiratory support and at least one consultant with more than 50\% of their time dedicated to neonatology) and low activity (<500 days and no dedicated neonatal consultant). Only level 3 services with units categorised as 'high' or 'medium' activity services were used in our analysis.

In this paper, an antenatal transfer was defined as any transfer into a level 3 service between booking and delivery; neonatal transfers were defined as those occurring within $24 \mathrm{~h}$ of birth. Later transfers, primarily for surgery or back transfer to the mother's local hospital, were not considered. Neonatal morbidity was defined as one or more of retinopathy of prematurity requiring retinal surgery, moderate or severe bronchopulmonary dysplasia (oxygen or respiratory support at 36 weeks' postmenstrual age), a severe brain injury (haemorrhagic parenchymal infarct, cystic changes or hydrocephalus on cerebral ultrasound) or necrotising enterocolitis managed by laparotomy.

\section{Statistical analysis}

The principal aim of this study was to determine the effect of the categorisation of the service providing care at birth and the influence of transfer before and after delivery. Only gestation and weight for gestation at birth, which are the most important determinants of survival, ${ }^{8}$ were used as confounders, unless otherwise stated.

The case mix of transferred mothers or babies is likely to be different from those who are not transferred. We addressed potential bias accruing from this in the following ways: analysis was first carried out comparing all babies born in level 3 services with all those born in level 2 services. This comparison was repeated after excluding births to women transferred antenatally to a level 3 service. We then compared the population of antenatal transfers to a level 3 service to the population born and remaining in level 2 or 3 services, respectively. This was repeated for neonatal transfers comparing all transfers to babies admitted to level 2 or 3 neonatal units but not transferred. We also considered outcomes for babies based on the hospital at which the woman first booked to have her care, which approximates to level 2 births plus antenatal transfers and may be considered to be a 'intention to deliver' analysis. For these analyses, crude and adjusted ORs and their 95\% CIs were calculated using logistic regression.

Time-specific mortality outcomes were assessed to determine whether the risk/benefits were similar at all stages of the clinical pathway. Using logistic regression, we calculated adjusted ORs (aORs) divided into deaths before delivery, delivery room deaths, deaths after neonatal unit admission before 7 days, between 7 and 28 days and after 28 days but before discharge. In each case, the aOR is based on the population still alive at the beginning of the time period under consideration. These analyses were structured as described in the previous paragraph.

We then evaluated bias that might occur from preferential treatment of multiple births by analysing only singleton pregnancies and the pattern of important determinants of perinatal
Figure 1 Population of births reported to the EPICure 2 study by place of birth and transfer status, indicating the population of babies included in antenatal transfer, neonatal transfer and activity analyses.

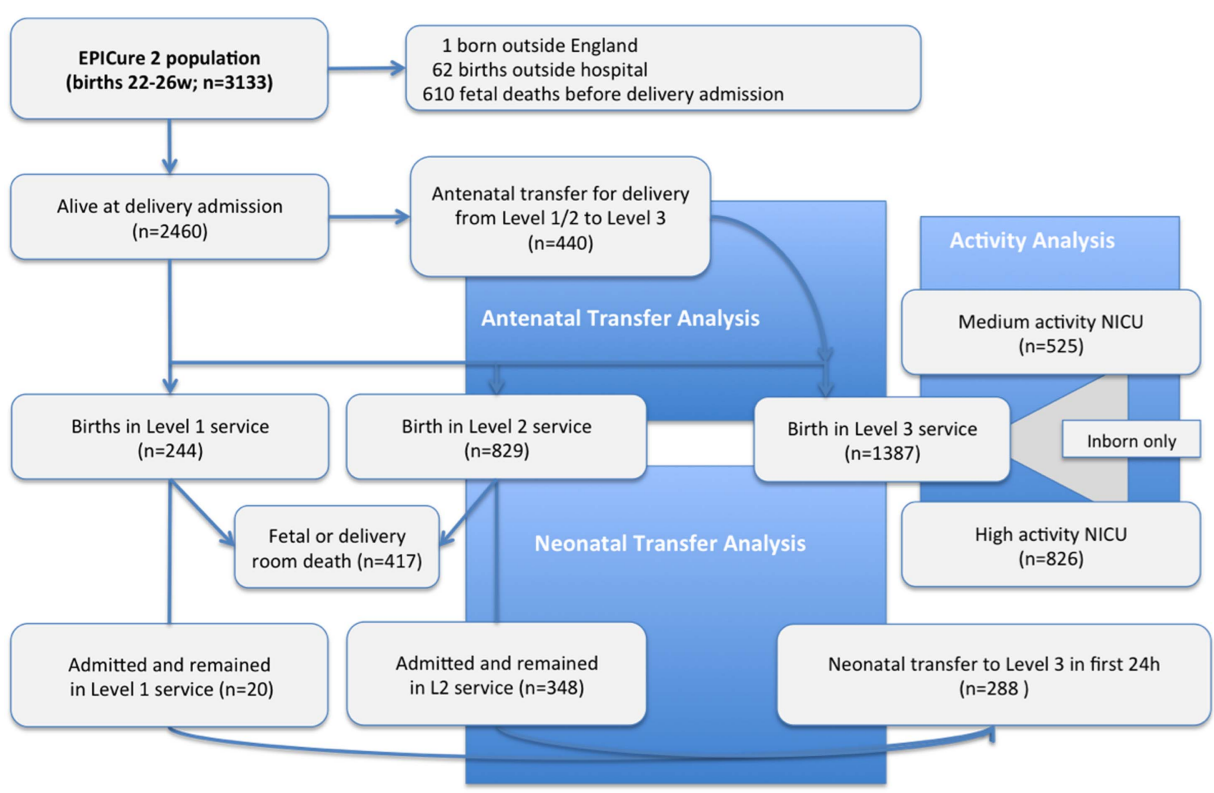


Table 1 Time-specific mortality and morbidity to discharge from hospital for all births in England in 2006 between 22 and 26 weeks of gestation

\begin{tabular}{|c|c|c|c|c|c|c|c|c|c|c|}
\hline \multirow{2}{*}{$\begin{array}{l}\text { Outcome } \\
\text { Antenatal death }\end{array}$} & \multicolumn{2}{|c|}{$\begin{array}{l}\text { Level } 1 \\
\text { services } \\
(n=244)\end{array}$} & \multicolumn{2}{|c|}{$\begin{array}{l}\text { Level } 2 \\
\text { services } \\
(n=829)\end{array}$} & \multicolumn{2}{|c|}{$\begin{array}{l}\text { Level } 3 \\
\text { services } \\
(n=1387)\end{array}$} & \multirow{2}{*}{$\begin{array}{l}\text { p Value* } \\
<0.0001\end{array}$} & \multicolumn{3}{|c|}{$\begin{array}{l}\text { Adjusted OR }(95 \% \mathrm{Cl}) \text { based on population } \\
\text { alive at start of time period }\end{array}$} \\
\hline & 77 & $32 \%$ & 174 & $21 \%$ & 208 & $15 \%$ & & & $0.81(0.63$ to 1.04$)$ & \\
\hline Delivery room deaths & 43 & $18 \%$ & 143 & $17 \%$ & 148 & $11 \%$ & $<0.001$ & & $0.55(0.39$ to 0.77$)$ & \\
\hline Neonatal unit deaths $<7$ days & 38 & $16 \%$ & 116 & $14 \%$ & 168 & $12 \%$ & $<0.0001$ & & $0.65(0.49$ to 0.86$)$ & \\
\hline All early neonatal deaths & 81 & $33 \%$ & 259 & $31 \%$ & 316 & $23 \%$ & $<0.0001$ & & $0.59(0.46$ to 0.75$)$ & \\
\hline Late neonatal death (7-28 days) & 12 & $5 \%$ & 56 & $7 \%$ & 122 & $9 \%$ & 0.999 & & $0.94(0.66$ to 1.33$)$ & \\
\hline Death 29 days to discharge & 6 & $2 \%$ & 35 & $4 \%$ & 86 & $6 \%$ & 0.976 & & $1.11(0.72$ to 1.72$)$ & \\
\hline \multirow[t]{2}{*}{ All deaths } & 176 & $72 \%$ & 524 & $63 \%$ & 732 & $53 \%$ & $<0.0001$ & & $0.73(0.59$ to 0.90$)$ & \\
\hline & & & & & & & & Alive on admission & Live births & Admission to Neonatal unit \\
\hline Survivors without morbidity $†$ & 19 & $8 \%$ & 70 & $8 \%$ & 157 & $11 \%$ & 0.017 & 1.27 (0.93 to 1.74$)$ & $1.25(0.91$ to 1.71$)$ & $1.24(0.90$ to 1.71$)$ \\
\hline \multicolumn{11}{|l|}{ Survivors only } \\
\hline Percent without morbidity $\dagger$ & & $28 \%$ & & $23 \%$ & & $24 \%$ & 0.769 & 1.13 (0.81 to 1.57$)$ & & \\
\hline
\end{tabular}

outcome: administration of antenatal steroids in order to reduce neonatal lung disease, no active intervention for the baby after birth, being alive with heart rate $>100$ at 5 min (excluding those with no active intervention), admission for neonatal care and (for admissions) the Clinical Risk Index for Babies II (CRIB-II) score. ${ }^{9}$

For each analysis, we also report the $\mathrm{p}$ value for a Wald test of the overall significance of place of birth, activity level, antenatal or postnatal transfer as appropriate. All statistical analyses were performed using Stata V.12 (StataCorp LP, College Station, Texas, USA).

\section{RESULTS}

Among the 182 hospitals with consultant-led obstetric services, 46 were reported to have level 3,84 level 2 and 52 level 1 neonatal units. Using the staffing and activity data collected for this study, 24 of the level 3 neonatal units were categorised as high activity, 21 as medium activity and 1 as low activity.

There were 2460 births at 22-26 weeks of gestation where the fetus was alive when the mother presented to hospital in her delivery admission, of which 244 (9.9\%) occurred in level 1 services, $829(33.7 \%)$ in level 2 services and 1387 (56.4\%) in level 3 services. In total, 382 women were transferred to level 3 services before birth (440 fetuses) and 288 babies were neonatal transfers within $24 \mathrm{~h}$ of birth (figure 1 ).

\section{Mortality}

Place of birth: Survival differed significantly by place of birth $(\mathrm{p}<0.0001)$. The proportion of stillbirths and delivery room deaths was lowest in level 3 services (23\%) and highest in level
Figure 2 Mortality by place of birth, perinatal transfer and high and medium activity level 3 services for babies born in England in 2006 at 26 completed weeks of gestation or less. $\mathrm{GA}$, gestational age in weeks; BW, birthweight for gestation as ' $z$ ' score; L2/L3, level of service; ANT, antenatal transfer; NNT, neonatal transfer; CRIB, clinical risk index for babies II score"; ANS, any antenatal steroid given to mother.

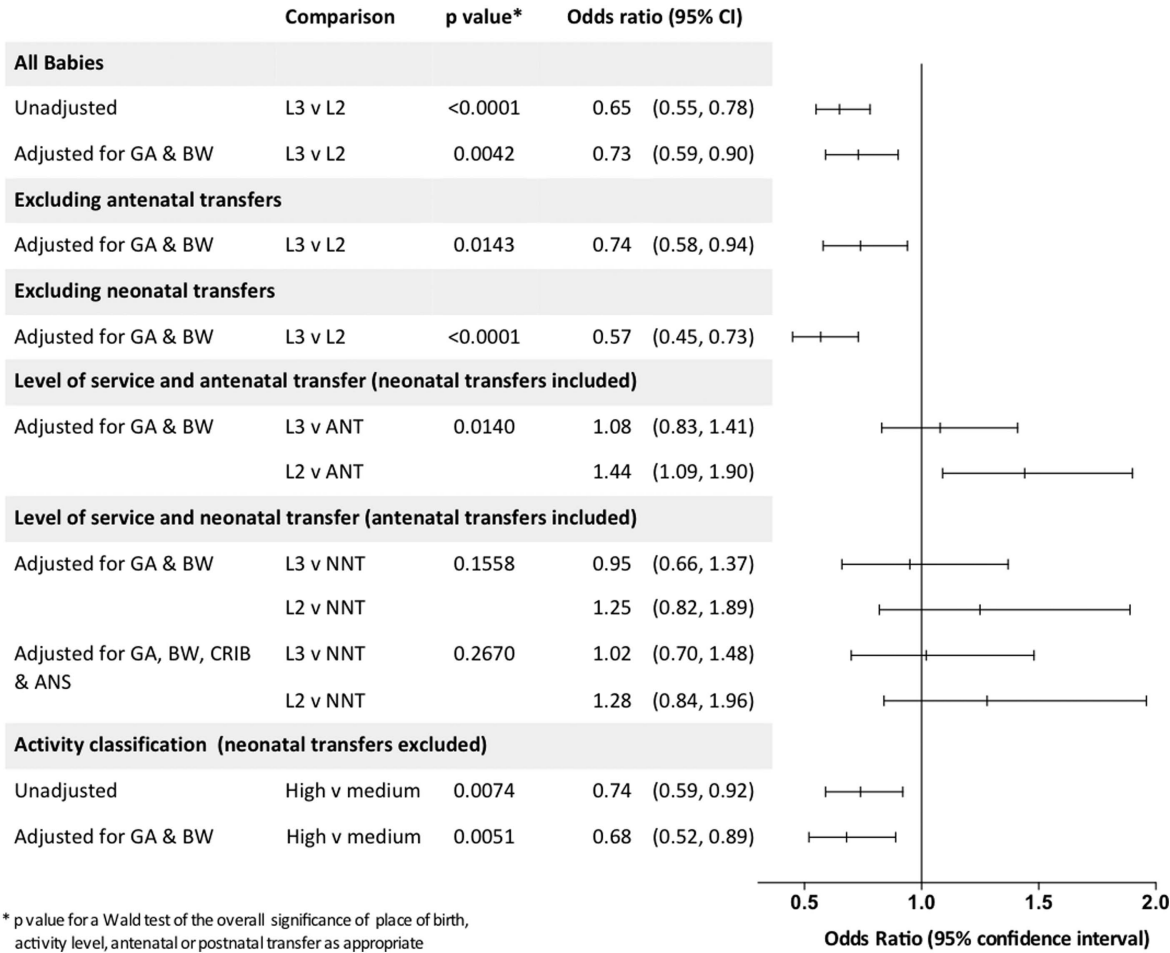



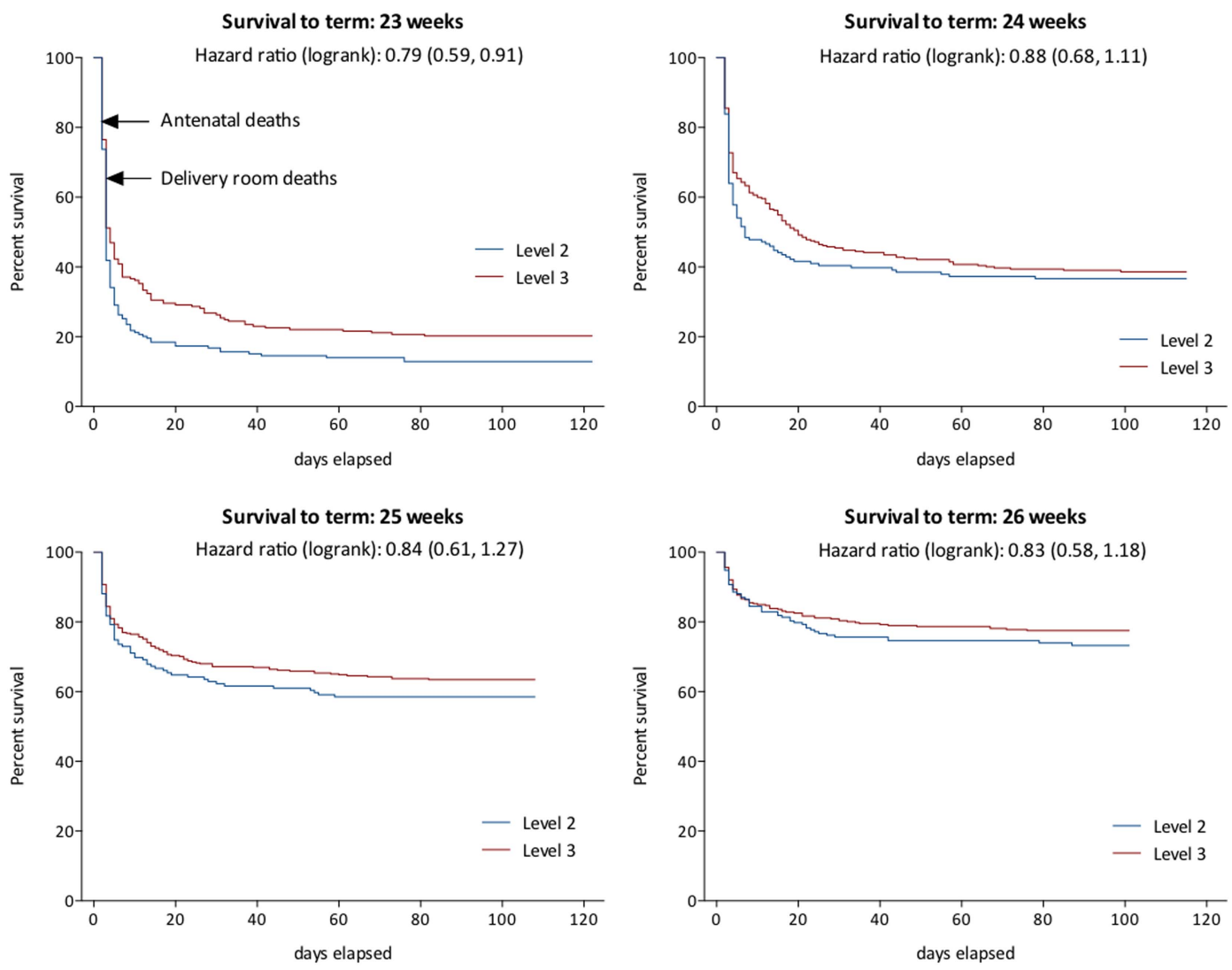

Figure 3 Kaplan-Meier plots of survival by gestational week for babies born at 23-26 weeks of gestation in level 3 and level 2 centres in England in 2006. Antenatal deaths are plotted as $t=1$ and delivery room outcomes care as $t=2$, with admission to neonatal unit as $t=3$ and subsequently postnatal age in days to 40 weeks postmenstrual age.

1 services (42\%; table 1$)$. The median age of death among neonatal unit admissions was earlier for births in level 1 services (median 2 days) compared with level 2 services (5 days) or level 3 services (10 days). Births in level 1 services were excluded from further analysis.

Overall mortality was lower in level 3 compared with level 2 services (OR 0.65 (95\% CI 0.55 to 0.78$)$ ), and following adjustment for gestational age and birthweight for gestation was only marginally attenuated (aOR $0.73(0.59$ to 0.90$)$ ). There were also few differences when babies that were transferred either antenatally or postnatally were excluded. Considering all babies but including transfer status (antenatal or postnatal) as a covariate in the model does not materially alter the mortality risk (figure 2).

Antenatal transfer: The transferred population had lower mortality compared with those who booked and were born in either level 3 or level 2 services (crude ORs 1.48 (1.26 to 1.75) and 2.21(1.87 to 2.61), respectively; $\mathrm{p}<0.0001$ for both). After adjustment, babies born to mothers who were not transferred from a level 2 service had an increased odds of death compared with those who were transferred (aOR 1.44 (1.09 to 1.90)). However, there was no difference in survival between those transferred antenatally into and those booked and born in level 3 services (aOR 1.08 (0.83 to 1.41); figure 2).

Hospital of booking: As the women who are transferred out may represent those with the highest chance of good outcomes, we considered the same population but allocated to the category of hospital where each woman booked for maternity care, as opposed to the hospital where delivery occurred. Babies of women booked in level 3 services still had reduced mortality compared with babies of women booked in level 2 services (aOR 0.79 (0.63 to 0.98$)$ ).

Neonatal transfer: No differences were found in survival for babies postnatally transferred compared with those who were born and remained in level 2 or level 3 services.

\section{Time-specific mortality outcomes}

Place of birth

Differences in survival for babies born in level 3 services occur early in the perinatal period (table 1 and figure 3 ). There were fewer deaths antenatally, in the delivery room and during the first 7 days after birth in level 3 compared with level 2 services; reflected in the proportions surviving to hospital discharge: $36.8 \%$ in level 2 services, and $47.2 \%$ in level 3 services. After adjustment, only fetal and early neonatal deaths (aOR 0.59 $(0.46$ to 0.75$))$ and overall mortality remained statistically significant.

\section{Antenatal transfer}

For women who were not transferred into a level 3 service, the adjusted odds for the fetus of antenatal death (aOR 1.52 (1.03 to 2.26)), overall early neonatal deaths (aOR 1.49 (1.10 to 2.02)) and deaths to discharge were significantly higher than for those who were transferred (table 2). There were no significant differences in outcomes for the transferred cases compared with those booked and delivered in hospitals with level 3 services.

\section{Postnatal transfer}

The results for postnatal transfer show few differences in mortality between neonatal transfer (NNT) and babies not 
Table 2 Mortality and morbidity to discharge for babies of women transferred antenatally and for those who delivered in level 2 and level 3 services without transfer for births in England in 2006 between 22 and 26 weeks of gestation

\begin{tabular}{|c|c|c|c|c|c|c|c|c|c|c|}
\hline Outcome & $\begin{array}{l}\text { Level } 2 \\
(n=829)\end{array}$ & & $\begin{array}{l}\text { Antenatal } \\
\text { transfer } \\
(n=440)\end{array}$ & & $\begin{array}{l}\text { Level } 3 \\
(n=947)\end{array}$ & & p Value* & $\begin{array}{l}\text { aOR } \\
\text { L3 vs L2 }\end{array}$ & $\begin{array}{l}\text { aOR } \\
\text { Level } 2 \text { v ANT }\end{array}$ & $\begin{array}{l}\text { aOR } \\
\text { Level } 3 \text { vs ANT }\end{array}$ \\
\hline Antenatal death & 174 & $21 \%$ & 45 & $10 \%$ & 163 & $17 \%$ & 0.104 & 0.86 (0.66 to 1.13$)$ & 1.52 (1.03 to 2.26$)$ & 1.31 (0.89 to 1.93$)$ \\
\hline Delivery room deathst & 143 & $17 \%$ & 30 & $7 \%$ & 118 & $12 \%$ & 0.002 & $0.53(0.37$ to 0.77$)$ & 1.67 (1.02 to 2.72$)$ & $0.89(0.54$ to 1.46$)$ \\
\hline Neonatal unit deaths $<7$ days & 116 & $14 \%$ & 50 & $11 \%$ & 118 & $12 \%$ & 0.005 & $0.69(0.51$ to 0.94$)$ & 1.80 (1.23 to 2.63$)$ & $1.25(0.86$ to 1.81$)$ \\
\hline All early neonatal deaths & 259 & $31 \%$ & 80 & $18 \%$ & 236 & $25 \%$ & 0.0001 & $0.61(0.47$ to 0.79$)$ & 1.85 (1.33 to 2.57$)$ & $1.12(0.81$ to 1.56$)$ \\
\hline Late neonatal death (7-28 days) $\dagger$ & 56 & $7 \%$ & 43 & $10 \%$ & 79 & $8 \%$ & 0.932 & 0.94 (0.64 to 1.38$)$ & 1.08 (0.69 to 1.68$)$ & 1.01 (0.67 to 1.53$)$ \\
\hline Death 29 days to discharge & 35 & $4 \%$ & 32 & $7 \%$ & 54 & $6 \%$ & 0.853 & $1.08(0.68$ to 1.73$)$ & $0.86(0.50$ to 1.46$)$ & $0.93(0.57$ to 1.51$)$ \\
\hline All deaths & 524 & $63 \%$ & 200 & $45 \%$ & 532 & $56 \%$ & 0.014 & 0.75 (0.59 to 0.95$)$ & 1.44 (1.09 to 1.90$)$ & $1.08(0.83$ to 1.41$)$ \\
\hline Survival without morbidity & 70 & $8 \%$ & 65 & $15 \%$ & 92 & $10 \%$ & 0.086 & $1.14(0.80$ to 1.61$)$ & $0.65(0.44$ to 0.96$)$ & $0.74(0.51$ to 1.06$)$ \\
\hline \multicolumn{11}{|l|}{ Survivors only } \\
\hline No morbidity & 70 & $23 \%$ & 65 & $27 \%$ & 92 & $22 \%$ & 0.166 & 0.99 (0.69 to 1.43$)$ & 0.72 (0.48 to 1.08 ) & 0.71 (0.49 to 1.04$)$ \\
\hline \multicolumn{11}{|l|}{ Perinatal factors } \\
\hline Antenatal steroid (any) & $531 / 824$ & $64 \%$ & $412 / 436$ & $95 \%$ & $656 / 936$ & $70 \%$ & $<0.0001$ & $1.15(0.90$ to 1.47$)$ & $0.20(0.12$ to 0.31$)$ & $0.23(0.14$ to 0.36$)$ \\
\hline Resuscitation withheld & $89 / 653$ & $14 \%$ & $12 / 395$ & $3 \%$ & $61 / 784$ & $8 \%$ & 0.002 & $0.41(0.25$ to 0.66$)$ & 1.25 (0.62 to 2.55$)$ & 0.51 (0.24 to 1.09$)$ \\
\hline Alive with $\mathrm{HR}>100$ at $5 \mathrm{~m}$ & $463 / 551$ & $84 \%$ & $342 / 379$ & $90 \%$ & $610 / 710$ & $86 \%$ & 0.243 & $1.16(0.84$ to 1.60$)$ & $0.70(0.46$ to 1.06$)$ & 0.81 (0.53 to 1.22$)$ \\
\hline Admitted to NNU & $511 / 829$ & $62 \%$ & $365 / 440$ & $83 \%$ & $664 / 947$ & $70 \%$ & 0.014 & 1.41 (1.09 to 1.81$)$ & $0.69(0.50$ to 0.96$)$ & $0.98(0.70$ to 1.35$)$ \\
\hline
\end{tabular}

transferred (table 3). Of note early neonatal deaths are more common in non-transferred babies admitted to level 2, implying perhaps that the sickest babies are not transferred.

\section{Survival without morbidity}

In contrast to mortality, neonatal morbidity does not appear to vary significantly with place of birth (table 1 ). The proportions of survivors without morbidity are similar for those born in hospitals with level 3 services (11\%) compared with those with level 2 (8\%), either considering the whole population $(11 \%$ vs $8 \%$, respectively) or only those who survived (23\% vs $24 \%$, respectively).

There was no evidence of an association between antenatal transfer and survival without morbidity compared with babies born in either level 2 or level 3 services, although the ORs were of a similar order in favour of antenatal transfer in each comparison (table 2). In contrast, babies born in centres with level 3 neonatal facilities have significantly higher odds of survival without morbidity compared with babies transferred in after birth (OR 1.92 (1.02 to 3.60)).
There was no evidence of significant variation in specific morbidities between groups (not shown).

\section{Activity in level 3 services}

For all mortality outcomes, unadjusted ORs were lower in highactivity units compared with medium activity, and the difference widened after adjustment for gestation and birthweight for gestation (table 4 and figure 2). There was no evidence for differences in time-specific mortality, nor was there any difference in survival without morbidity in high-throughput units compared with low-throughput units ( $\mathrm{aOR} 0.79(0.55$ to 1.14$)$ ) or in the proportion surviving without morbidity among survivors (aOR $0.69(0.47$ to 1.02$))$.

\section{Further analyses}

Using data only from singleton pregnancies reflected the whole population analysis and did not alter our conclusions (see online supplementary tables S1 and S2).

We next investigated whether there were differences in prespecified determinants of outcome.

Table 3 Mortality and morbidity to discharge for neonatal unit admissions for babies who were transferred to a level 3 service after admission (NNT) compared with babies who remained in their hospital of birth, for births in England in 2006 between 22 and 26 weeks of gestation

\begin{tabular}{|c|c|c|c|c|c|c|c|c|c|c|}
\hline \multirow{2}{*}{$\begin{array}{l}\text { Outcome } \\
\text { Neonatal unit deaths }<7 \text { days }\end{array}$} & \multicolumn{2}{|c|}{$\begin{array}{l}\text { Level } 2 \\
(\mathrm{n}=348)(\%)\end{array}$} & \multicolumn{2}{|c|}{$\begin{array}{l}\text { Neonatal } \\
\text { transfer } \\
(n=261)\end{array}$} & \multicolumn{2}{|c|}{$\begin{array}{l}\text { Level } 3 \\
(n=1028)\end{array}$} & \multirow{2}{*}{$\begin{array}{l}\text { p Value* } \\
0.002\end{array}$} & \multirow{2}{*}{$\begin{array}{l}\text { aOR L3 vs L2 } \\
0.56(0.41 \text { to } 0.78)\end{array}$} & \multirow{2}{*}{$\begin{array}{l}\text { aOR L2 vs NNT } \\
1.50(0.93 \text { to } 2.42)\end{array}$} & \multirow{2}{*}{$\begin{array}{l}\text { aOR L3 vs NNT } \\
0.85(0.55 \text { to } 1.30)\end{array}$} \\
\hline & 80 & $23 \%$ & 35 & $21 \%$ & 166 & $16 \%$ & & & & \\
\hline Late neonatal death (7-28 days) & 34 & 10 & 22 & $14 \%$ & 122 & $12 \%$ & 0.935 & $0.93(0.61$ to 1.43$)$ & $1.01(0.55$ to 1.86$)$ & 0.94 (0.56 to 1.58$)$ \\
\hline Death 29 days to discharge & 22 & 6 & 13 & $8 \%$ & 86 & $8 \%$ & 0.831 & 1.05 (0.63 to 1.76$)$ & 1.16 (0.54 to 2.49$)$ & 1.22 (0.64 to 2.34$)$ \\
\hline All deaths & 136 & $39 \%$ & 70 & $43 \%$ & 374 & $36 \%$ & 0.156 & 0.76 (0.58 to 1.00$)$ & 1.25 (0.82 to 1.89$)$ & 0.95 (0.66 to 1.37$)$ \\
\hline Survival without morbidity & 58 & $17 \%$ & 12 & $7 \%$ & 157 & $15 \%$ & 0.124 & 1.09 (0.77 to 1.54$)$ & $1.76(0.90$ to 3.46$)$ & $1.92(1.02$ to 3.60$)$ \\
\hline \multicolumn{11}{|l|}{ Survivors only } \\
\hline No morbidity & 58 & $27 \%$ & 12 & $13 \%$ & 157 & $24 \%$ & 0.172 & 0.97 (0.67 to 1.40$)$ & $1.88(0.94$ to 3.78$)$ & $1.83(0.96$ to 3.50$)$ \\
\hline
\end{tabular}


Table 4 Mortality and morbidity to discharge for babies cared for in level 3 services categorised by medium and high-activity levels (see text)

\begin{tabular}{|c|c|c|c|c|c|c|}
\hline \multirow{2}{*}{$\begin{array}{l}\text { Outcome } \\
\text { Antenatal death }\end{array}$} & \multicolumn{2}{|c|}{$\begin{array}{l}\text { High activity } \\
(\mathrm{n}=826)\end{array}$} & \multicolumn{2}{|c|}{$\begin{array}{l}\text { Medium activity } \\
(n=525)\end{array}$} & \multirow{2}{*}{$\begin{array}{l}\text { aOR high vs medium activity } \\
0.92 \text { (0.66 to } 1.27)\end{array}$} & \multirow{2}{*}{$\frac{p \text { Value* }}{0.600}$} \\
\hline & 121 & $15 \%$ & 85 & $16 \%$ & & \\
\hline Delivery room deaths* & 82 & $10 \%$ & 65 & $12 \%$ & 0.78 (0.49 to 1.22 ) & 0.274 \\
\hline Neonatal unit deaths $<7$ days & 94 & $11 \%$ & 68 & $13 \%$ & $0.82(0.58$ to 1.17$)$ & 0.275 \\
\hline All early neonatal deaths & 176 & $21 \%$ & 133 & $25 \%$ & 0.78 (0.58 to 1.06$)$ & 0.119 \\
\hline Late neonatal death (7-28 days) * & 69 & $8 \%$ & 50 & $10 \%$ & $0.72(0.47$ to 1.08$)$ & 0.115 \\
\hline Death 29 days to discharge & 49 & $6 \%$ & 35 & $7 \%$ & 0.65 (0.40 to 1.06$)$ & 0.086 \\
\hline All deaths & 415 & $50 \%$ & 303 & $58 \%$ & $0.68(0.52$ to 0.89$)$ & 0.005 \\
\hline Survival without neonatal morbidity & 86 & $10 \%$ & 65 & $12 \%$ & 0.79 (0.55 to 1.14$)$ & 0.205 \\
\hline \multicolumn{7}{|l|}{ Survivors only } \\
\hline No neonatal morbidity & 86 & $21 \%$ & 65 & $29 \%$ & $0.69(0.47$ to 1.02$)$ & 0.063 \\
\hline
\end{tabular}

Overall, antenatal steroid was administered to $45 \%$ of births in hospitals with level 1, 64\% in hospitals with level 2 and $78 \%$ in hospitals with level 3 services. The odds of receiving steroid are 2.5 times higher for each week of gestational age (OR 2.50 (95\% CI 2.29 to 2.72$)$ ), controlling for service category, and 1.83 times (95\% CI 1.58 to 2.12) higher for each increase in service level, controlling for gestational age.

Planned no active intervention was recorded in $15 \%$ of births in level 1, 14\% in level 2 and $6 \%$ in level 3 services. The odds of there being no active intervention are 0.62 (95\% CI 0.47 to 0.82 ) for each change in service category, controlling for gestational age.

When comparing births in level 2 services with those transferred antenatally, mothers less frequently received steroid, a plan for no active intervention was more frequent and the baby was less likely to have a heart rate $>100 \mathrm{bpm}$ at $5 \mathrm{~min}$ (table 2). Antenatal transfers were also more likely to receive steroid compared with non-transferred level 3 births. CRIB-II scores were not significantly different between groups (not shown), indicating similarity of the condition of the babies on admission for neonatal care.

When we considered hospital of booking, babies of women booked in level 3 services were less likely to have resuscitation withheld (aOR 0.48 ( 0.29 to 0.79$)$ ) and more likely to be admitted for neonatal intensive care (aOR 1.31 (1.02 to 1.68)), but they had similar rates of steroid administration and similar chances of being alive with a heart rate $>100 \mathrm{bpm}$ at $5 \mathrm{~min}$ (aOR 0.97 (0.76 to 1.24 ) and 1.08 (0.79 to 1.48$)$, respectively).

\section{DISCUSSION}

These national data represent the first study of outcomes for the entire perinatal service since neonatal care in England was reorganised into managed clinical networks. Within this reorganisation there has been much local debate as to the appropriate setting in which to care for extremely preterm infants. We have demonstrated that in 2006 only $56.4 \%$ of extremely preterm births occurred in the recommended setting of level 3 services and that birth in such a service was associated with reduced mortality, particularly among deaths occurring around delivery and during the first 7 days after birth. Considering the population of women booked in level 3 services, greater survival is also apparent, suggesting that service differences may be important. Furthermore, differences in survival between high and medium neonatal activity levels in level 3 services emphasise the importance of the setting in which care takes place.

Whereas survival increased between 1995 and 2006 in babies born between 22 and 25 weeks of gestation, neonatal morbidity, in terms of brain injury, bronchopulmonary dysplasia, retinopathy and severe necrotising enterocolitis, was unchanged. ${ }^{1}$ In this study, the place of birth and perinatal transfer had little impact on the proportion with morbidities in any setting. It appears likely that reducing morbidity in this population may require different strategies from those that have successfully enhanced survival.

\section{Potential limitations}

Comparisons such as this are fraught with difficulty. In particular, the data may be confounded by differences in case mix, mainly of gestational age and birthweight for gestation, consequent on local demography, women's choice of place of birth and both antenatal and postnatal transfers. For this reason, we have reported comparisons adjusted for gestation and weight for gestation at birth and, while we have no information about whether women with health problems or previous adverse pregnancy outcomes preferentially 'booked' in level 3 services, because of the detail in the EPICure database we are able to account for all transfers thereafter. Because we had recorded the place of booking in the pregnancy, we were also able to correct for bias that may occur with the transfer out of good prognosis pregnancies and confirm reduced mortality for babies whose mothers booked in level 3 services.

It was not possible to determine how many of the births in level 1 or level 2 services could have been avoided if there had been a more active approach to the initiation of corticosteroid therapy and arranging antenatal transfer as soon as extremely preterm birth was expected. Some births in level 2 services occurred either because the mother arrived in hospital at too advanced a stage of labour, with a complication preventing her safe transfer, or because at 22 or 23 weeks prognosis was considered too poor to warrant active intervention. Women who present to the labour ward and rapidly deliver are likely to be those who receive least steroid and thus have worse neonatal early lung disease and worse outcomes. Using outcomes based on hospital of booking, we see no differences in the rate of steroid use between women booked in level 2 and level 3 services, confirming the bias for those not transferred. 
Furthermore, management was more active following antenatal transfer, perhaps because there was sufficient time for safe transfer and there were higher expectations of a good outcome. Our data support the safety of antenatal transfer for the baby.

\section{Comparisons with other studies}

Most published comparisons, ${ }^{4}$ including data from the UK, ${ }^{6} 7$ use neonatal unit admissions as their denominator population, thereby effectively ignoring policy and competency concerning perinatal and immediate delivery room care. In these comparisons, many use case mix correction strategies, such as CRIB-II, ${ }^{9}$ to correct for different practices and outcomes in the delivery room. In our study, for babies admitted to neonatal units, CRIB-II scores are not significantly different between babies admitted in level 2 and level 3 neonatal units. In contrast, our findings suggest that care in the perinatal period is particularly important in reducing mortality and are consistent with our reported observation that improvements in mortality of admitted babies between 1995 and 2006 relate to improved mortality around delivery and during the first week after birth. ${ }^{1}$

An analysis of routinely collected data from neonatal admissions in England has shown increased postnatal transfers of babies born at 27 and 28 weeks of gestation in 2009 and 2010 compared with data for babies of the same gestation born between 1998 and 2000 before regionalisation of services. ${ }^{10}$ It was notable when comparing the 2006 EPICure cohort of admissions born before 26 weeks with the previous cohort born in $1995,{ }^{1}$ that there had been an increase in the proportion transferred within $24 \mathrm{~h}$ of birth. However, postnatal transfer in 2006 was not associated with increased morbidity as it had been in 1995, probably associated with the introduction of networkbased dedicated neonatal transfer teams.

In different health services outside the UK, there is evidence that centralised services produce better survival outcomes for babies requiring neonatal intensive care. A recent systematic review used data from the USA (22 studies) and from the rest of the world (15 studies, including studies from Canada, Israel, Australia and Europe, but not the UK). Overall the odds of survival in level 3 compared with level 2 services were 1.60 (1.33 to 1.92 ), slightly higher in studies of babies $<1000 \mathrm{~g}$ birthweight $(1.80(1.31$ to 2.46$))$ than in very preterm infants $(1.42$ (1.06 to 1.88$))^{4}$

Our study is based upon the population for whom such transfer is recommended in current guidance. ${ }^{11}$ Even after excluding births at 22 weeks, at which gestation active intervention is not recommended under current guidance, ${ }^{12}$ and at 26 weeks, for whom currently transfer is not universal, still only $59 \%$ of births at 23-25 weeks of gestation are born in recommended settings. In contrast, among liveborn babies born in the Parisian region of France between 24 and 31 weeks of gestation, between 1997 and 2003, 77\% of births occurred in level 3 centres; inpatient mortality and the prevalence of intraventricular haemorrhage were significantly lower in such centres, while the rate of periventricular leucomalacia and bronchopulmonary dysplasia remained constant. ${ }^{13}$

\section{CONCLUSIONS}

In this whole population study, we have demonstrated improved neonatal survival for babies born between 22 and 26 weeks of gestation and managed in level 3 services in England, and further improvements in survival for those babies managed in level 3 intensive care units with higher levels of activity. These data support the concept that hospital expertise and activity are important factors in improved outcomes for high-risk pregnancies with threatened extremely preterm birth. ${ }^{14} 15$ Recent UK data confirm that neonatal nurse staffing levels continue to be associated with quality of care, ${ }^{16}$ but many of our level 3 services are smaller than those associated with improved outcomes. ${ }^{17}$ Despite this, our successful networks are currently at risk in the reorganisation of the NHS. ${ }^{18}$ We speculate that further development of these structures with larger and appropriately staffed neonatal services integrated with maternity networks is required if survival for extremely preterm babies is to continue to improve.

Acknowledgements The EPICure outcome studies were sponsored by the University of Nottingham (till September 2008) and subsequently by University College London. NM receives part funding from the Department of Health's NIHR Biomedical Research Centre's funding scheme at UCLH/UCL

Contributors NM and KLC formulated the hypothesis and NM, EMH, ASM and CB the design of the analysis. $\mathrm{CB}$ analysed the data under supervision from $\mathrm{EMH}$ and NM. Perinatal data were collated by KLC, ESD and EMH. NM wrote the first draft and coordinated the manuscript. All authors were involved in interpretation of the data and writing the report. All have seen and approved the final version.

Oversight EPICure Studies Steering Committee Independent Members: Professor Peter Brocklehurst (Chairman), Mrs Jane Abbott, Professor Andrew Bush, Professor Richard Cooke, Mrs Noreen Maconochie, Mrs Alison Matthews, Mr David Matthews, Dr Richard Morton, Dr Maggie Redshaw, Professor David Taylor, Mr Nigel Turner, Mrs Diane Turner and Mr Patrick Walsh.

Research governance The Medical Research Council funded the study in full (G0401525) and had no role in study design, data collection, data analysis, data interpretation, or writing of the report. City and East London REC 3 granted ethical approval for the EPICure 2 study (05/Q0605/107).

Funding Medical Research Council.

\section{Competing interests None.}

Ethics approval City and East London REC 3.

Provenance and peer review Not commissioned; externally peer reviewed.

Data sharing statement The EPICure Studies have a formal data sharing policy available from http://www.epicure.ac.uk

Open Access This is an Open Access article distributed in accordance with the Creative Commons Attribution Non Commercial (CC BY-NC 3.0) license, which permits others to distribute, remix, adapt, build upon this work non-commercially, and license their derivative works on different terms, provided the original work is properly cited and the use is non-commercial. See: http://creativecommons.org/ licenses/by-nc/3.0/

\section{REFERENCES}

1 Costeloe KL, Hennessy EM, Haider S, et al. Short term outcomes after extreme preterm birth in England: comparison of two birth cohorts in 1995 and 2006 (the EPICure studies). BMJ 2012;345:e7976.

2 Moore T, Hennessy EM, Myles J, et al. Neurological and developmental outcome in extremely preterm children born in England in 1995 and 2006: the EPICure studies. BMJ 2012;345:e7961.

3 Marlow N, Bryan Gill A. Establishing neonatal networks: the reality. Arch Dis Child Fetal Neonatal Ed 2007;92:F137-42.

4 Lasswell SM, Barfield WD, Rochat RW, et al. Perinatal regionalization for very low-birth-weight and very preterm infants: a meta-analysis. JAMA 2010;304: 992-1000.

5 Department of Health. Report of DH Expert Working Group on neonatal intensive care services. London: Department of Health, 2003.

6 Selinger M. Benefits of reorganising neonatal and obstetric services are unclear. BMJ 2012;344:e3203.

7 Tucker J, Tarnow-Mordi W, Gould C, et al. UK neonatal intensive care services in 1996. On behalf of the UK Neonatal Staffing Study Collaborative Group. Arch Dis Child Fetal Neonatal Ed 1999:80:F233-4.

8 Tyson JE, Parikh NA, Langer J, et al. Intensive care for extreme prematuritymoving beyond gestational age. NEJM 2008;358:1672-81.

9 Parry G, Tucker J, Tarnow-Mordi W, et al. CRIB II: an update of the clinical risk index for babies score. Lancet 2003;361:1789-91.

10 Gale C, Santhakumaran S, Nagarajan S, et al. Impact of managed clinical networks on NHS specialist neonatal services in England: population based study. BMJ 2012;344:e2105.

11 Department of Health. Toolkit for High Quality Neonatal Services. London: Department of Health, 2009. 
12 Wilkinson AR, Ahluwalia J, Cole A, et al. Management of babies born extremely preterm at less than 26 weeks of gestation: a framework for clinical practice at the time of birth. Arch Dis Child Fetal Neonatal Ed 2009;94:F2-5.

13 Zeitlin J, Ancel PY, Delmas D, et al. Changes in care and outcome of very preterm babies in the Parisian region between 1998 and 2003. Arch Dis Child Fetal Neonatal Ed 2010;95:F188-93.

14 Chung JH, Phibbs CS, Boscardin WJ, et al. Examining the effect of hospital-level factors on mortality of very low birth weight infants using multilevel modeling. J Perinatol 2011;31:770-5.
15 Finnstrom 0 , Olausson PO, Sedin G, et al. The Swedish national prospective study on extremely low birthweight (ELBW) infants. Incidence, mortality, morbidity and survival in relation to level of care. Acta Paediatrica 1997:86:503-11.

16 Pillay T, Nightingale P, Owen $S$, et al. Neonatal nurse staffing and delivery of clinica care in the SSBC Newborn Network. Arch Dis Child Fetal Neonatal Ed 2012;97: F174-8.

17 Phibbs CS. Managed clinical networks in neonatal care. BMJ 2012;344:e2423.

18 Boyle R. Clinical networks are effective, work in patients' interests, and shouldn't be disbanded. BMJ 2013;346:f565. 